\title{
A Description of Transverse Momentum Distributions in $p+p$ Collisions at RHIC and LHC Energies
}

\author{
Jia-Qi Hui and Zhi-Jin Jiang (iD \\ College of Science, University of Shanghai for Science and Technology, Shanghai 200093, China \\ Correspondence should be addressed to Zhi-Jin Jiang; jzj265@163.com
}

Received 2 January 2019; Revised 22 March 2019; Accepted 14 April 2019; Published 5 May 2019

Guest Editor: Raghunath Sahoo

Copyright ( 2019 Jia-Qi Hui and Zhi-Jin Jiang. This is an open access article distributed under the Creative Commons Attribution License, which permits unrestricted use, distribution, and reproduction in any medium, provided the original work is properly cited. The publication of this article was funded by $\mathrm{SCOAP}^{3}$.

\begin{abstract}
It has long been debated whether the hydrodynamics is suitable for the smaller colliding systems such as $p+p$ collisions. In this paper, by assuming the existence of longitudinal collective motion and long-range interactions in the hot and dense matter created in $p+p$ collisions, the relativistic hydrodynamics incorporating with the nonextensive statistics is used to analyze the transverse momentum distributions of the particles. The investigations of the present paper show that the hybrid model can give a good description of the currently available experimental data obtained in $p+p$ collisions at RHIC and LHC energies, except for $p$ and $\bar{p}$ produced in the range of $p_{T}>3.0 \mathrm{GeV} / \mathrm{c}$ at $\sqrt{s}=200 \mathrm{GeV}$.
\end{abstract}

\section{Introduction}

In the past decade, the experimental results of heavy ion collisions at both RHIC and LHC energies have been extensively studied. These studies have shown that the strongly coupled quark-gluon plasma (sQGP) might be created in these collisions [1-9], which exhibits a clear collective behavior almost like a perfect fluid with very low viscosity [1028]. Therefore, the evolution of sQGP can be described in the scope of relativistic hydrodynamics. However, unlike heavy ion collisions, $p+p$ collisions are a relatively smaller system with lower multiplicity, larger viscosity, and larger fluctuation [29]. The reasonableness of applying relativistic hydrodynamics in depicting the evolution of sQGP created in $p+p$ collisions has undergone an endless debate.

In this paper, by supposing the existence of collective flow in $p+p$ colliding direction, the relativistic hydrodynamics including phase transition is introduced to describe the longitudinal expansion of sQGP. Besides the collective flow, the thermal motion also exists in sQGP. The evolution of sQGP is therefore the superposition of collective flow and thermal motion. Known from the investigations of [30, 31], the long-range interactions and memory effects might appear in sQGP. This guarantees the reasonableness of nonextensive statistics in describing the thermodynamic aspects of sQGP. Hence, in this paper, we will use the nonextensive statistics instead of conventional statistics to characterize the thermal motion of the matter created in $p+p$ collisions.

The nonextensive statistics, i.e., Tsallis nonextensive thermostatistics, is the generalization of conventional Boltzmann-Gibbs statistics, which is proposed by C. Tsallis in his pioneer work of [32]. This statistical theory overcomes the inabilities of the conventional statistical mechanics by assuming the existence of long-range interactions, longrange microscopic memory, or fractal space-time constraints in the thermodynamic system. It has a wide range of applications in cosmology [33], phase shift analyses for the pion-nucleus scattering [34], dynamical linear response theory, and variational methods [35]. It has achieved a great success in solving many physical problems, such as the solar neutrino problems [36], many-body problems, the problems in astrophysical self-gravitating systems [37], and the transverse momentum spectra [38-40].

The article is organized as follows. In Section 2, a brief description is given about the employed hydrodynamics, presenting its analytical solutions. The solutions are then used in Section 3 to formulate the transverse momentum distributions of the particles produced in $p+p$ collisions in 
the light of Cooper-Frye prescription. The last Section 4 is about conclusions.

\section{A Brief Introduction to the Hydrodynamic Model}

The main content of the relativistic hydrodynamic model [15, 41] used in this paper is as follows.

The expansion of fluid obeys the continuity equation

$$
\partial_{\mu} T^{\mu \nu}=0, \quad \mu, v=0,1,
$$

where

$$
T^{\mu \nu}=(\varepsilon+p) u^{\mu} u^{\nu}-p g^{\mu \nu}
$$

is the energy-momentum tensor of fluid and $g^{\mu \nu}=$ $\operatorname{diag}(1,-1)$ is the metric tensor. The four-velocity of fluid $u^{\mu}=\left(u^{0}, u^{1}\right)=\left(\cosh y_{F}, \sinh y_{F}\right)$, where $y_{F}$ is the rapidity of fluid. $\varepsilon$ and $p$ in Equation (2) are the energy density and pressure of fluid, respectively, which are related by the sound speed $c_{s}$ of fluid via the equation of state

$$
\frac{\mathrm{d} p}{\mathrm{~d} \varepsilon}=\frac{s \mathrm{~d} T}{T \mathrm{~d} s}=c_{s}^{2}
$$

where $T$ and $s$ are the temperature and entropy density of fluid, respectively.

The projection of Equation (1) to the direction of $u_{\mu}$ leads to the continuity equation for entropy conservation

$$
\partial_{v}\left(s u^{v}\right)=0
$$

The projection of Equation (1) to the direction perpendicular to $u_{\mu}$ gives equation

$$
\frac{\partial\left(T \sinh y_{F}\right)}{\partial t}+\frac{\partial\left(T \cosh y_{F}\right)}{\partial z}=0
$$

which means the existence of a scalar function $\phi$ satisfying

$$
\begin{aligned}
& \frac{\partial \phi}{\partial t}=T \cosh y_{F} \\
& \frac{\partial \phi}{\partial z}=-T \sinh y_{F} .
\end{aligned}
$$

By using $\phi$ and Legendre transformation, Khalatnikov potential $\chi$ can be introduced via relation

$$
\chi=\phi-t T \cosh y_{F}+z T \sinh y_{F},
$$

which changes the coordinate base of $(t, z)$ to that of $\left(\omega, y_{F}\right)$

$$
\begin{aligned}
& t=\frac{e^{\omega}}{T_{0}}\left(\frac{\partial \chi}{\partial \omega} \cosh y_{F}+\frac{\partial \chi}{\partial y_{F}} \sinh y_{F}\right), \\
& z=\frac{e^{\omega}}{T_{0}}\left(\frac{\partial \chi}{\partial \omega} \sinh y_{F}+\frac{\partial \chi}{\partial y_{F}} \cosh y_{F}\right),
\end{aligned}
$$

where $T_{0}$ is the initial temperature of sQGP, and $\omega=-\ln (T /$ $T_{0}$ ). In terms of $\chi$, Equation (4) can be rewritten as the socalled equation of telegraphy

$$
\frac{\partial^{2} \chi}{\partial \omega^{2}}-2 \beta \frac{\partial \chi}{\partial \omega}-\frac{1}{c_{s}^{2}} \frac{\partial^{2} \chi}{\partial y_{F}^{2}}=0, \quad \beta=\frac{1-c_{s}^{2}}{2 c_{s}^{2}}
$$

With the expansion of created matter, its temperature becomes lower and lower. When the temperature drops from the initial temperature $T_{0}$ to the critical temperature $T_{c}$, phase transition occurs. This will modify the value of sound speed of fluid. In sQGP, $c_{s}=c_{0}=1 / \sqrt{3}$, which is the sound speed of a massless perfect fluid, being the maximum of $c_{s}$. In the hadronic state, $0<c_{s}=c_{h} \leq c_{0}$. At the point of phase transition, $c_{s}$ is discontinuous.

The solutions of Equation (10) for sQGP and hadronic state are, respectively [15],

$$
\begin{aligned}
& \chi_{0}\left(\omega, y_{F}\right)=\frac{Q_{0} c_{0}}{2} e^{\beta_{0} \omega} I_{0}\left(\beta_{0} \sqrt{\omega^{2}-c_{0}^{2} y_{F}^{2}}\right), \\
& \chi_{h}\left(\omega, y_{F}\right)=\frac{Q_{0} c_{0}}{2} S(\omega) I_{0}\left[\lambda\left(\omega, y_{F}\right)\right],
\end{aligned}
$$

where $I_{0}$ is the 0th order modified Bessel function, and

$$
\begin{gathered}
\beta_{0}=\frac{\left(1-c_{0}^{2}\right)}{2 c_{0}^{2}}=1, \\
S(\omega)=e^{\beta_{h}\left(\omega-\omega_{c}\right)+\beta_{0} \omega_{c}}, \\
\lambda\left(\omega, y_{F}\right)=\beta_{h} c_{h} \sqrt{y_{h}^{2}(\omega)-y_{F}^{2}},
\end{gathered}
$$

where $\beta_{h}=\left(1-c_{h}^{2}\right) / 2 c_{h}^{2}, \omega_{c}=-\ln \left(T_{c} / T_{0}\right)$, and $y_{h}(\omega)=$ $\left[\left(\omega-\omega_{c}\right) / c_{h}\right]+\left(\omega_{c} / c_{0}\right)$. The $Q_{0}$ in Equations (11) and (12) is a free parameter determined by fitting the theoretical results with experimental data.

\section{The Transverse Momentum Distributions of the Particles Produced in $p+p$ Collisions}

3.1. The Energy of Quantum of Produced Matter. The nonextensive statistics is based on the following two postulations $[32,36]$. of

(a) The entropy of a statistical system possesses the form

$$
s_{q}=\frac{1}{q-1}\left(1-\sum_{i=1}^{\Omega} p_{i}^{q}\right)
$$

where $p_{i}$ is the probability of a given microstate among $\Omega$ ones and $q$ is a fixed real parameter. The defined entropy has the usual properties of positivity, equiprobability, and irreversibility, and, in the limit of $q \longrightarrow 1$, it reduces to the conventional Boltzmann-Gibbs entropy

$$
s=-\sum_{i} p_{i} \ln p_{i}
$$


(b) The mean value of an observable $\mathcal{O}$ is defined as

$$
\overline{\mathcal{O}}_{q}=\sum_{i=1}^{\Omega} p_{i}^{q} \mathcal{O}_{i}
$$

where $\mathcal{O}_{i}$ is the value of an observable $\mathcal{O}$ in the microstate $i$.

From the above two postulations, the average occupational number of quantum in the state with temperature $T$ can be written in a simple analytical form [42]

$$
\bar{n}_{q}=\frac{1}{\left[1+(q-1)\left(E-\mu_{B}\right) / T\right]^{1 /(q-1)}+\delta} .
$$

Here, as usual, $E$ is the energy of quantum, and $\mu_{B}$ is its baryochemical potential. For baryons $\delta=1$ and for mesons $\delta=-1$. In the limit of $q \longrightarrow 1$, it reduces to the conventional Fermi-Dirac or Bose-Einstein distributions. Hence, the value of $q$ in the nonextensive statistics represents the degree of deviation from the conventional statistics. Known from Equation (17), the average energy of quantum in the state with temperature $T$ reads

$$
\begin{aligned}
& \bar{E}_{q} \\
& =\frac{m_{T} \cosh \left(y-y_{F}\right)}{\left\{1+\left[(q-1)\left(m_{T} \cosh \left(y-y_{F}\right)-\mu_{B}\right)\right] / T\right\}^{1 /(q-1)}+\delta},
\end{aligned}
$$

where $y$ is the rapidity of quantum and $m_{T}=\sqrt{p_{T}^{2}+m^{2}}$ is its transverse mass with rest mass $m$ and transverse momentum $p_{T}$.

3.2. The Transverse Momentum Distributions of the Particles Produced in $p+p$ Collisions. With the expansion of hadronic matter, its temperature becomes even lower. As the temperature drops to the so-called kinetic freeze-out temperature $T_{f}$, the inelastic collisions among hadronic matter stop. The yields of produced particles remain unchanged, becoming the measured results. According to Cooper-Frye scheme [43], the invariant multiplicity distributions of produced particles take the form $[15,43]$

$$
\begin{aligned}
& \frac{d^{2} N}{2 \pi p_{T} d y d p_{T}}=\frac{A}{(2 \pi)^{3}} \\
& \left.\cdot \int_{-y_{h}\left(\omega_{f}\right)}^{y_{h}\left(\omega_{f}\right)}\left(\cosh y \frac{d z}{d y_{F}}-\sinh y \frac{d t}{d y_{F}}\right) \bar{E}_{q}\right|_{T=T_{f}} d y_{F},
\end{aligned}
$$

where $A$ is the area of overlap region of collisions, $\omega_{f}=$ $-\ln \left(T_{f} / T_{0}\right)$, and the integrand takes values at the moment of $T=T_{f}$. The meaning of Equation (19) is evident. The part of integrand in the round brackets is proportional to the rapidity density of fluid [43]. Hence, Equation (19) is the convolution of rapidity of fluid with the energy of the particles in the state with temperature T. From Equations (8) and (9)

$$
\begin{gathered}
\cosh y \frac{d z}{d y_{F}}-\sinh y \frac{d t}{d y_{F}} \\
=\frac{1}{T} c_{s}^{2} \frac{\partial}{\partial \omega}\left(\chi+\frac{\partial \chi}{\partial \omega}\right) \cosh \left(y-y_{F}\right) \\
-\frac{1}{T} \frac{\partial}{\partial y_{F}}\left(\chi+\frac{\partial \chi}{\partial \omega}\right) \sinh \left(y-y_{F}\right) .
\end{gathered}
$$

Substituting $\chi$ in Equation (20) by the $\chi_{h}$ of Equation (12) and taking the values at the moment of $T=T_{f}$, it becomes

$$
\begin{aligned}
& \left.\left(\cosh y \frac{d z}{d y_{F}}-\sinh y \frac{d t}{d y_{F}}\right)\right|_{T=T_{f}}=\frac{Q_{0} c_{0}}{T_{f}}\left(\beta_{h} c_{h}\right)^{2} \\
& \cdot S\left(\omega_{f}\right)\left[B\left(\omega_{f}, y_{F}\right) \sinh \left(y-y_{F}\right)\right. \\
& \left.+C\left(\omega_{f}, y_{F}\right) \cosh \left(y-y_{F}\right)\right],
\end{aligned}
$$

where

$$
\begin{gathered}
B\left(\omega_{f}, y_{F}\right) \\
=\frac{\beta_{h} y_{F}}{\lambda\left(\omega_{f}, y_{F}\right)}\left\{\frac{\beta_{h} c_{h} y_{h}\left(\omega_{f}\right)}{\lambda\left(\omega_{f}, y_{F}\right)} I_{0}\left[\lambda\left(\omega_{f}, y_{F}\right)\right]\right. \\
\left.+\left[\frac{\beta_{h}+1}{\beta_{h}}-\frac{2 \beta_{h} c_{h} y_{h}\left(\omega_{f}\right)}{\lambda^{2}\left(\omega_{f}, y_{F}\right)}\right] I_{1}\left[\lambda\left(\omega_{f}, y_{F}\right)\right]\right\}, \\
C\left(\omega_{f}, y_{F}\right)=\left\{\frac{\beta_{h}+1}{\left.\beta_{h}+\frac{\left[\beta_{h} c_{h} y_{h}\left(\omega_{f}\right)\right]^{2}}{\lambda^{2}\left(\omega_{f}, y_{F}\right)}\right\}}\right. \\
\quad \cdot I_{0}\left[\lambda\left(\omega_{f}, y_{F}\right)\right]+\frac{1}{\lambda\left(\omega_{f}, y_{F}\right)}\left\{\frac{y_{h}\left(\omega_{f}\right)}{c_{h}}+1\right. \\
\left.-\frac{2\left[\beta_{h} c_{h} y_{h}\left(\omega_{f}\right)\right]^{2}}{\lambda^{2}\left(\omega_{f}, y_{F}\right)}\right\} I_{1}\left[\lambda\left(\omega_{f}, y_{F}\right)\right],
\end{gathered}
$$

where $\lambda\left(\omega_{f}, y_{F}\right)=\beta_{h} c_{h} \sqrt{y_{h}^{2}\left(\omega_{f}\right)-y_{F}^{2}}, I_{1}$ is the 1st order modified Bessel function.

By using Equations (19) and (21)-(23), we can obtain the transverse momentum distributions of produced particles as shown in Figures 1, 2, 3, and 4.

Figure 1 shows the transverse momentum spectra of $K_{\mathrm{S}}^{0}$, $K^{+}, K^{-}, \Lambda, \bar{\Lambda}, \Xi^{-}, \bar{\Xi}^{+}$, and $\Omega^{-}+\bar{\Omega}^{+}$produced in $p+p$ collisions at $\sqrt{s}=200 \mathrm{GeV}$. The solid dots, circles, and solid triangles represent the experimental data of the STAR Collaboration [44]. The solid curves are the results calculated from Equation (19). The values of free parameters $q, Q_{0}$, and $\chi^{2} / \mathrm{NDF}$ are listed in Table 1. It can be seen that the present model can give a good description of the transverse momentum distributions of strange particles. Since strangeness enhancement is originally proposed as a signature of sQGP produced in nuclear 

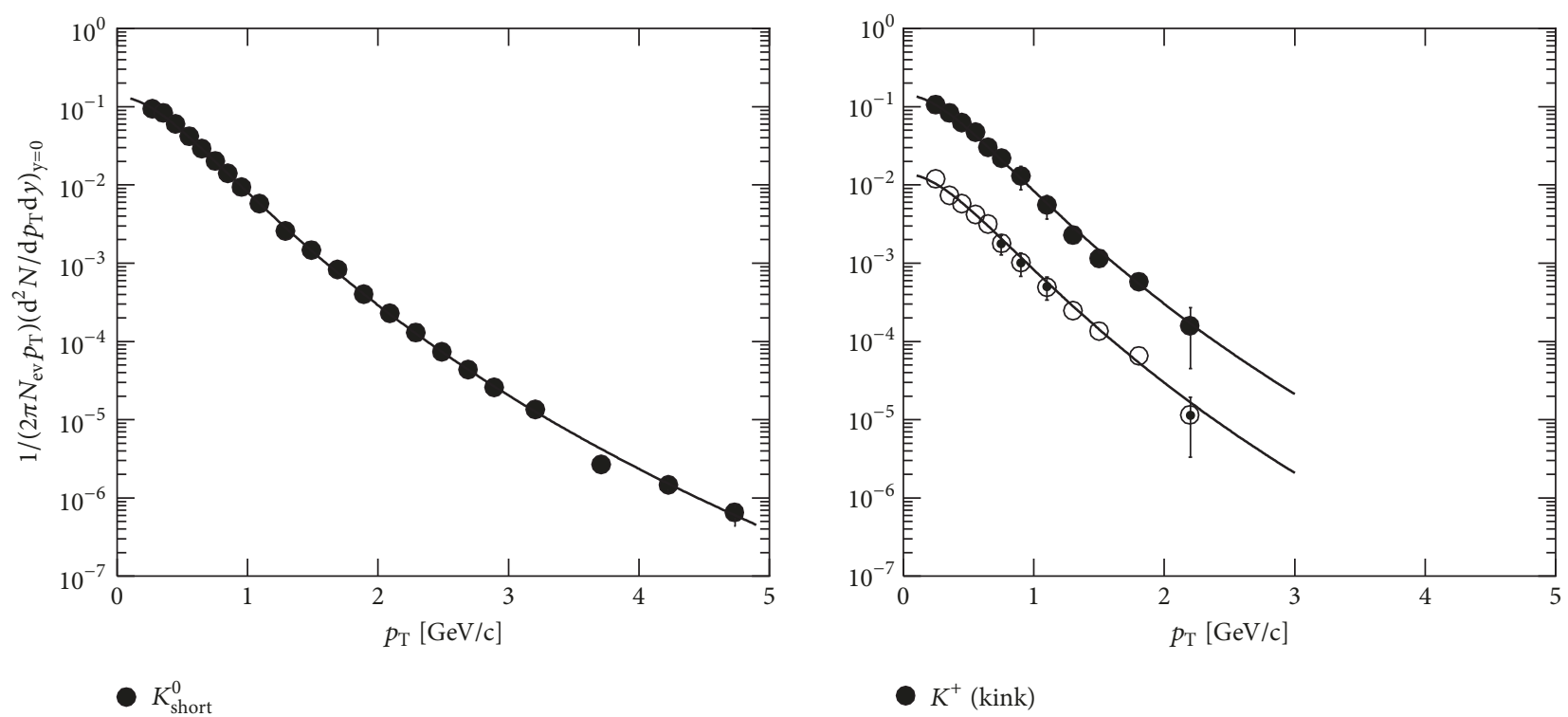

- $K^{+}$(kink)

$K^{-} / 10$ (kink)
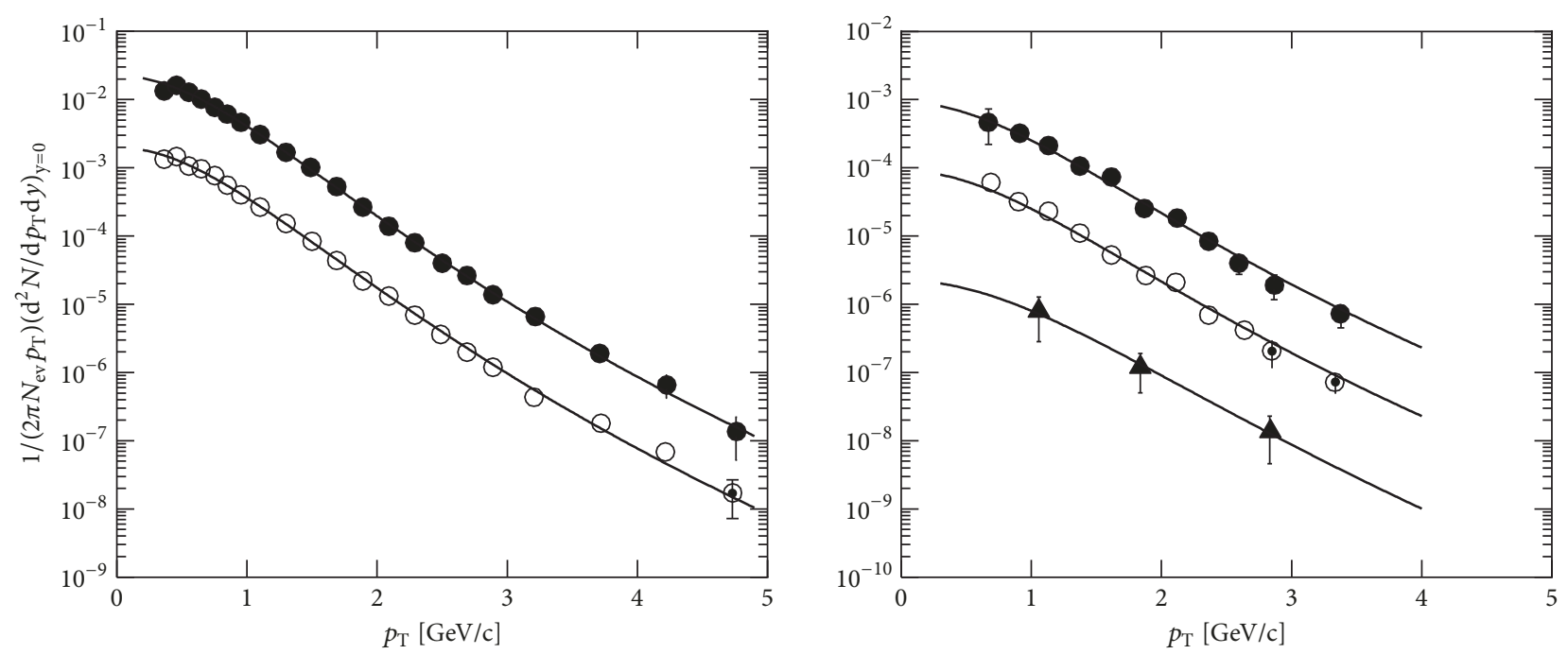

- $\Lambda$

- $\Xi^{-}$

$\bigcirc \bar{\Lambda} / 10$

○ $\bar{\Xi}^{+} / 10$

$\Delta \bar{\Omega}+\Omega / 50$

FIGURE 1: The transverse momentum distributions of strange particles $(|y|<0.5)$ produced in $p+p$ collisions at $\sqrt{s}=200 \mathrm{GeV}$. The solid dots, circles, and solid triangles represent the experimental data of the STAR Collaboration [44]. The solid curves are the results calculated from Equation (19).

TABLE 1: The values of $q, Q_{0}$, and $\chi^{2} / \mathrm{NDF}$ obtained from the analyses of STAR data [44] in $p+p$ collisions at $\sqrt{s}=200 \mathrm{GeV}$.

\begin{tabular}{lccccc}
\hline Parameters & $K_{\mathrm{S}}^{0}$ & $K^{+} / K^{-}$ & $\Lambda / \bar{\Lambda}$ & $\Xi^{-} / \bar{\Xi}^{+}$ & $1.075 \pm 0.003$ \\
\hline$q$ & $1.083 \pm 0.002$ & $1.083 \pm 0.005$ & $1.062 \pm 0.001$ & $1.075 \pm 0.003$ & $1.068 \pm 0.007$ \\
& & $1.083 \pm 0.006$ & $1.062 \pm 0.001$ & $0.026 \pm 0.006$ & $0.016 \pm 0.010$ \\
$Q_{0}$ & $0.084 \pm 0.005$ & $0.087 \pm 0.012$ & $0.379 \pm 0.026$ & $0.026 \pm 0.005$ & $0.47 / 0.64$ \\
$\chi^{2} / \mathrm{NDF}$ & 0.68 & $0.086 \pm 0.012$ & $0.337 \pm 0.023$ & $0.47 / 0.90$ & 0.02 \\
\hline
\end{tabular}




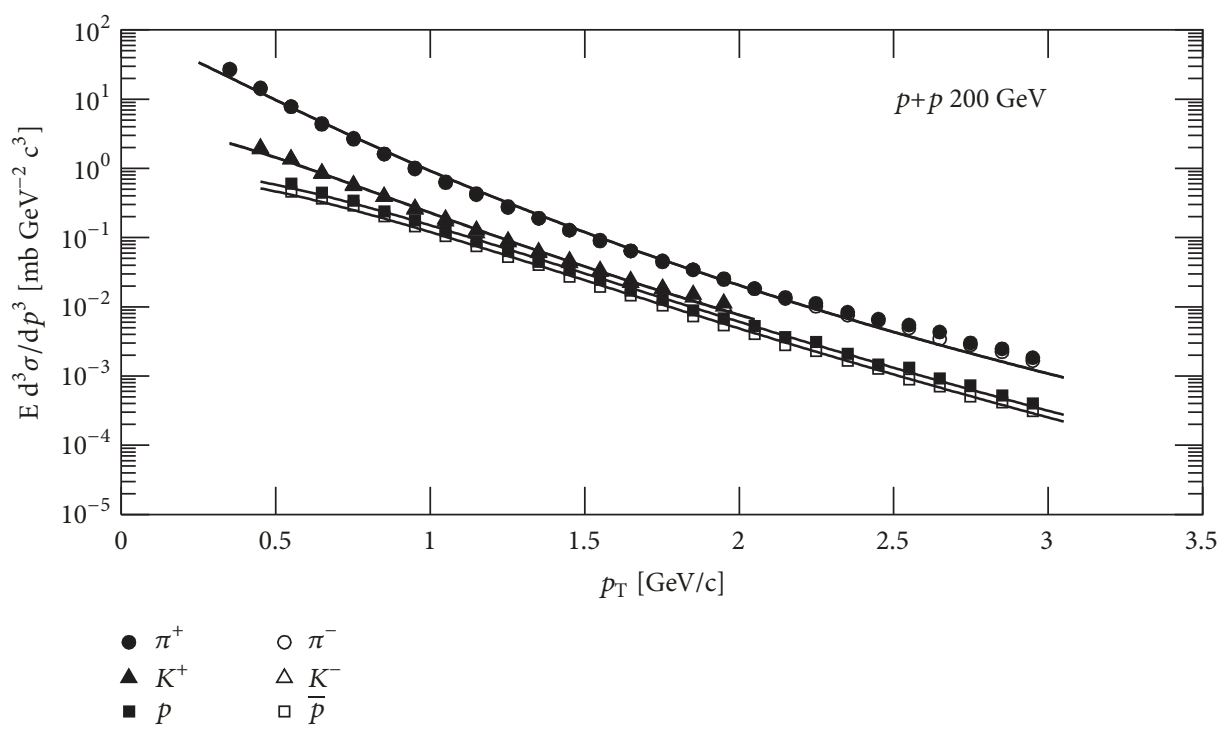

Figure 2: The transverse momentum distributions of $\pi^{+}, \pi^{-}, K^{+}, K^{-}, p$, and $\bar{p}$ produced in $p+p$ collisions at $\sqrt{s}=200 \mathrm{GeV}$ at midrapidity. The solid dots, solid triangles, solid squares, circles, triangles, and squares represent the experimental data of the PHENIX Collaboration [45]. The solid curves are the results calculated from Equation (19).

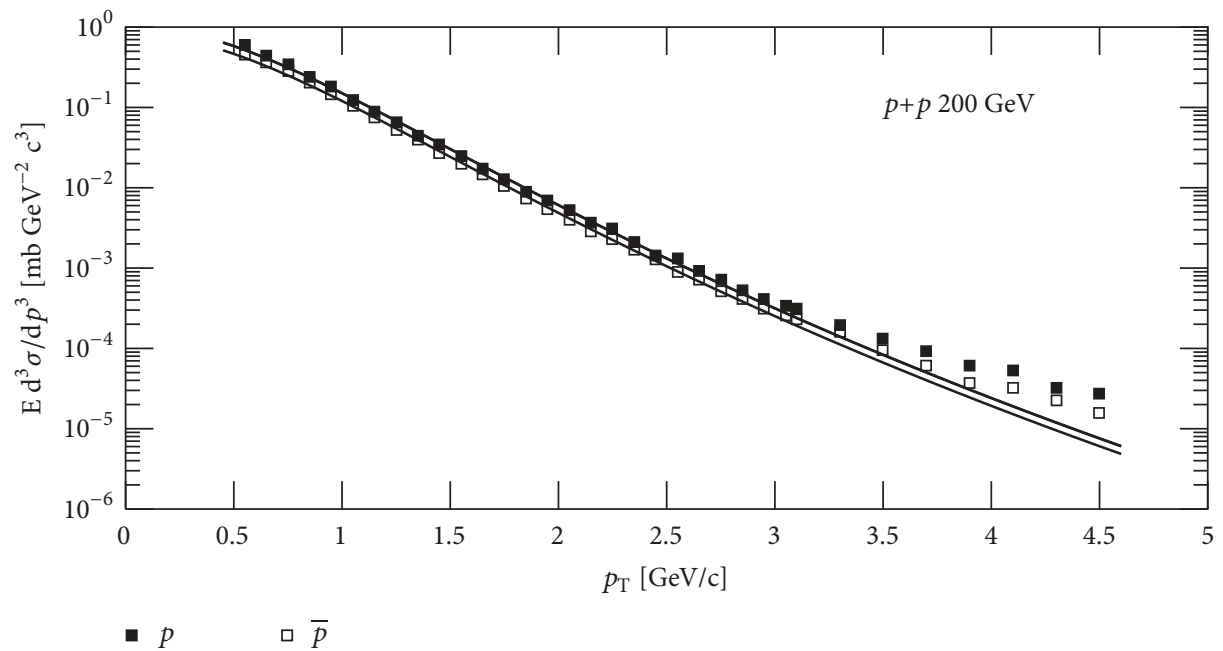

Figure 3: The transverse momentum distributions of $p$ and $\bar{p}$ produced in $p+p$ collisions at $\sqrt{s}=200 \mathrm{GeV}$ in the whole measured $p_{T}$ range. The solid squares and squares represent the experimental data of the PHENIX Collaboration [45]. The solid curves are the results calculated from Equation (19).

collisions, this proves the reasonableness of hypothesis given at the beginning of this paper that $\mathrm{QQGP}$ might appear in $p+p$ collisions.

Figure 2 presents the transverse momentum spectra of $\pi^{+}, \pi^{-}, K^{+}, K^{-}, p$, and $\bar{p}$ produced in $p+p$ collisions at $\sqrt{s}=200 \mathrm{GeV}$. The solid dots, solid triangles, solid squares, circles, triangles, and squares represent the experimental data of the PHENIX Collaboration [45]. The solid curves are the results calculated from Equation (19). The values of free parameters $q, Q_{0}$, and $\chi^{2} / \mathrm{NDF}$ are summarized in Table 2 . The theoretical model can give a good description of the experimental data for $\pi^{+}, \pi^{-}, K^{+}, K^{-}$in the whole measured transverse momentum range, and for $p$ and $\bar{p}$ in the range of
$p_{T} \leq 3.0 \mathrm{GeV} / \mathrm{c}$. In the range of $p_{T}>3.0 \mathrm{GeV} / \mathrm{c}$, the deviation appears as shown in Figure 3, which shows the transverse momentum distributions of $p$ and $\bar{p}$ in the whole measured $p_{T}$ range.

Figure 4 shows the transverse momentum spectra of $\pi^{+}$, $\pi^{-}, K^{+}, K^{-}, p$, and $\bar{p}$ produced in $p+p$ collisions at $\sqrt{s}=0.9$, 2.76 , and $7 \mathrm{TeV}$. The solid dots, solid triangles, solid squares, circles, triangles, and squares represent the experimental data of the CMS Collaboration [46]. The solid curves are the results calculated from Equation (19). The values of free parameters $q, Q_{0}$, and $\chi^{2} / \mathrm{NDF}$ are summarized in Table 3.

In calculations, the sound speed in hadronic state takes the value of $c_{h}=0.35[47,48]$. The critical temperature takes 

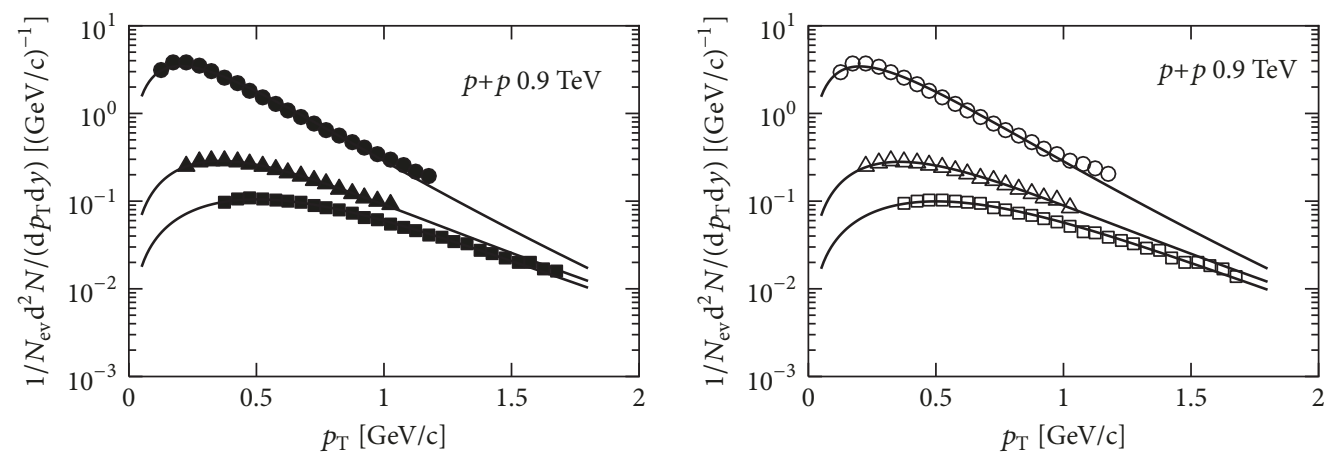

- $\pi^{+}$

- $p$

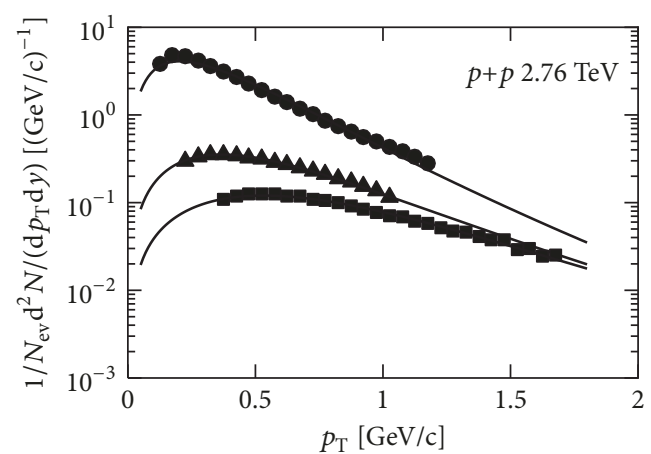

$\pi^{+}$

$\Delta K^{+}$

- $p$

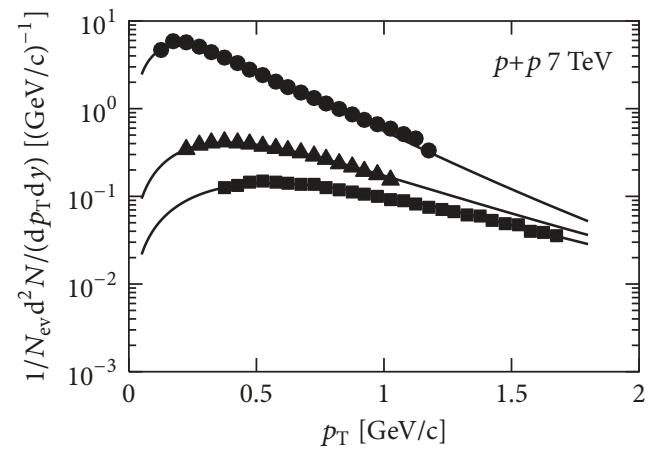

$\pi^{+}$

$\Delta K^{+}$

- $p$ $\circ \pi^{-}$

$\triangle K^{-}$

$\square \bar{p}$

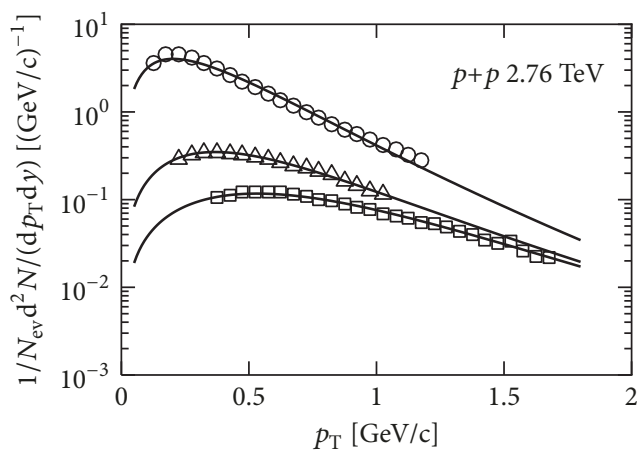

$\circ \pi^{-}$

$\triangle K^{-}$

$\square \bar{p}$

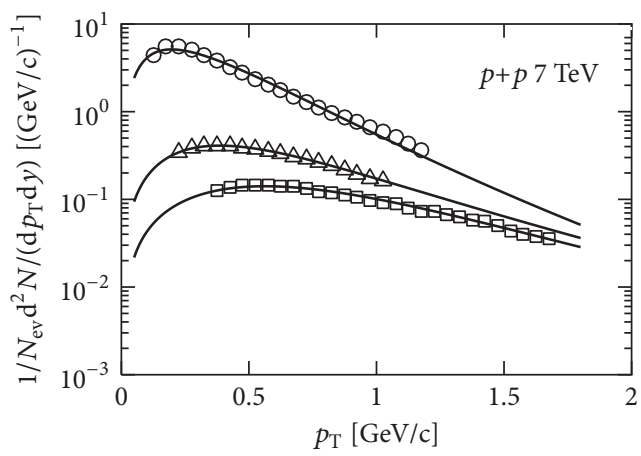

○ $\pi^{-}$

$\triangle K^{-}$

ㅁ $\bar{p}$

FIGURE 4: The transverse momentum distributions of the identified charged particles $(|y|<1)$ produced in $p+p$ collisions at $\sqrt{s}=0.9,2.76$, and $7 \mathrm{TeV}$ (from top to bottom). The solid dots, solid triangles, solid squares, circles, triangles, and squares represent the experimental data of the CMS Collaboration [46]. The solid curves are the results calculated from Equation (19).

TABLE 2: The values of $q, Q_{0}$, and $\chi^{2} / \mathrm{NDF}$ obtained from the analyses of PHENIX data [45] in $p+p$ collisions at $\sqrt{s}=200 \mathrm{GeV}$.

\begin{tabular}{lccr}
\hline Parameters & $\pi^{+} / \pi^{-}$ & $K^{+} / K^{-}$ & $p / \bar{p}$ \\
\hline$q$ & $1.075 \pm 0.003$ & $1.080 \pm 0.003$ & $1.060 \pm 0.002$ \\
& $1.075 \pm 0.003$ & $1.080 \pm 0.003$ & $1.060 \pm 0.001$ \\
$Q_{0}$ & $10.439 \pm 0.015$ & $3.699 \pm 0.005$ & $13.099 \pm 1.551$ \\
$\chi^{2} / \mathrm{NDF}$ & $10.342 \pm 0.015$ & $3.602 \pm 0.006$ & $10.479 \pm 1.221$ \\
\hline
\end{tabular}


TABLE 3: The values of $q, Q_{0}$, and $\chi^{2} / \mathrm{NDF}$ obtained from the analyses of CMS data [46] in $p+p$ collisions at LHC energies.

\begin{tabular}{|c|c|c|c|c|}
\hline$\sqrt{s}$ & Parameters & $\pi^{+} / \pi^{-}$ & $K^{+} / K^{-}$ & $p / \bar{p}$ \\
\hline \multirow{5}{*}{$0.9 \mathrm{TeV}$} & \multirow{2}{*}{$q$} & $1.064 \pm 0.002$ & $1.090 \pm 0.002$ & $1.071 \pm 0.001$ \\
\hline & & $1.064 \pm 0.003$ & $1.090 \pm 0.002$ & $1.071 \pm 0.001$ \\
\hline & \multirow{2}{*}{$Q_{0}$} & $0.161 \pm 0.004$ & $0.045 \pm 0.001$ & $0.152 \pm 0.005$ \\
\hline & & $0.159 \pm 0.004$ & $0.044 \pm 0.002$ & $0.145 \pm 0.003$ \\
\hline & $\chi^{2} / \mathrm{NDF}$ & $8.50 / 10.85$ & $0.37 / 0.47$ & $1.10 / 1.84$ \\
\hline \multirow{5}{*}{$2.76 \mathrm{TeV}$} & \multirow{2}{*}{$q$} & $1.078 \pm 0.002$ & $1.100 \pm 0.002$ & $1.088 \pm 0.001$ \\
\hline & & $1.078 \pm 0.002$ & $1.100 \pm 0.002$ & $1.088 \pm 0.001$ \\
\hline & \multirow{2}{*}{$Q_{0}$} & $0.051 \pm 0.001$ & $0.015 \pm 0.0004$ & $0.037 \pm 0.001$ \\
\hline & & $0.050 \pm 0.001$ & $0.014 \pm 0.0004$ & $0.036 \pm 0.001$ \\
\hline & $\chi^{2} / \mathrm{NDF}$ & $8.90 / 8.25$ & $0.30 / 0.62$ & $1.75 / 1.35$ \\
\hline \multirow{5}{*}{$7 \mathrm{TeV}$} & \multirow{2}{*}{$q$} & $1.084 \pm 0.002$ & $1.120 \pm 0.003$ & $1.105 \pm 0.001$ \\
\hline & & $1.084 \pm 0.003$ & $1.120 \pm 0.002$ & $1.105 \pm 0.001$ \\
\hline & \multirow{2}{*}{$Q_{0}$} & $0.004 \pm 0.00004$ & $0.001 \pm 0.00002$ & $0.002 \pm 0.00004$ \\
\hline & & $0.004 \pm 0.00008$ & $0.001 \pm 0.00002$ & $0.002 \pm 0.00002$ \\
\hline & $\chi^{2} / \mathrm{NDF}$ & 7.28/7.69 & $0.23 / 0.35$ & $1.13 / 1.43$ \\
\hline
\end{tabular}

the value of $T_{c}=0.16 \mathrm{GeV}$ [49]. For $\sqrt{s}=200 \mathrm{GeV}$, the initial temperature takes the value of $T_{0}=0.35 \mathrm{GeV}[50]$, the kinetic freeze-out temperature takes the values of $T_{f}=$ $0.12 \mathrm{GeV}$ for strange particles and pions, and, for protons, $T_{f}=0.13 \mathrm{GeV}$ from the investigation of [51], which also shows that the baryochemical potential takes the value of $\mu_{B}=0.01$ $\mathrm{GeV}$. For $\sqrt{s}=0.9,2.76$, and $7 \mathrm{TeV}$, referring to [50], the initial temperatures are estimated to be $T_{0}=0.4,0.6$, and $1.5 \mathrm{GeV}$, respectively. The kinetic freeze-out temperature takes the values of $T_{f}=0.12 \mathrm{GeV}$ for pions and kaons, and, for protons, $T_{f}=0.13 \mathrm{GeV}$. The baryochemical potential takes the value of $\mu_{B}=0$ [51].

The parameters $Q_{0}$ and $T_{0}$ have the same effects. They all affect the amplitudes of the theoretical curves. They are different from parameter $q$ which affects the slopes of the theoretical curves. From the above analysis we can see that the value of the parameter $q$ increases with the increase of the CMS beam energy. However, the values of $q$ do not seem completely consistent with the CMS and the RHIC beam energies.

\section{Conclusions}

By assuming the existence of longitudinal collective motion and long-range interactions in sQGP produced in $p+p$ collisions, the relativistic hydrodynamics including phase transition together with the nonextensive statistics is used to discuss the transverse momentum distributions of the particles produced in $p+p$ collisions at $\sqrt{s}=0.2,0.9,2.76$, and $7 \mathrm{TeV}$.

The theoretical model used in this paper contains rich information about the transport coefficients of fluid, such as the sound speed $c_{0}$ in sQGP, the sound speed $c_{h}$ in hadronic state, the initial temperature $T_{0}$, the critical temperature $T_{c}$, the kinetic freeze-out temperature $T_{f}$, and the baryochemical potential $\mu_{B}$. Except for $T_{0}$, the other five parameters take the values either from the widely accepted theoretical results or from experimental measurements. As for $T_{0}$, there are no acknowledged values so far. In this paper, $T_{0}$ takes the values from other studies. The investigations of the present paper show the conclusions as follows.

(a) The theoretical model can give a good description of the currently available experimental data collected in $p+p$ collisions at RHIC and LHC energies with the only exception of $p$ and $\bar{p}$ measured in the range of $p_{T}>3.0 \mathrm{GeV} / \mathrm{c}$ at $\sqrt{s}=200 \mathrm{GeV}$, which might be caused by the hard scattering process [52]. To improve the fitting conditions, the results of perturbative QCD should be taken into account.

(b) The fitted values of $q$ are close to 1 . This means that the deviation between nonextensive statistics and conventional statistics is small, while it is this small difference that plays an essential role in fitting the experimental data.

\section{Data Availability}

The experimental data used to support the findings of this study have been deposited in https://doi.org/ 10.1103/PhysRevC.75.064901; https://doi.org/10.1103/PhysRevC.83.064903; and https://doi.org/10.1140/epjc/s10052012-2164-1.

\section{Conflicts of Interest}

The authors declare that there are no conflicts of interest regarding the publication of this paper.

\section{Acknowledgments}

This work is supported by the Shanghai Key Lab of Modern Optical System.

\section{References}

[1] I. Arsene, I. G. Bearden, and D. Beavis, "Quark-gluon plasma and color glass condensate at RHIC? The perspective from the 
BRAHMS experiment," Nuclear Physics A, vol. 757, no. 1-2, pp. 1-27, 2005.

[2] B. B. Back, M. D. Baker, and M. Ballintijn, "The PHOBOS perspective on discoveries at RHIC," Nuclear Physics A, vol. 757, no. 1-2, pp. 28-101, 2005.

[3] J. Adams, M. M. Aggarwal, and Z. Ahammed, "Experimental and theoretical challenges in the search for the quark-gluon plasma: The STAR Collaboration's critical assessment of the evidence from RHIC collisions," Nuclear Physics A, vol. 757, no. 1-2, pp. 102-183, 2005.

[4] K. Adcox, S. S. Adler, S. Afanasiev et al., "RHIC: Experimental evaluation by the PHENIX Collaboration," Nuclear Physics A, vol. 757, no. 1-2, pp. 184-283, 2005.

[5] T. Alexopoulos et al., "Mass-identified particle production in proton-antiproton collisions at $\sqrt{s}=300,540,1000$, and 1800 GeV," Physical Review D, vol. 48, Article ID 984, 1993.

[6] P. Lévai and B. Müller, "Transverse baryon flow as possible evidence for a quark-gluon-plasma phase," Physical Review Letters, vol. 67, no. 12, pp. 1519-1522, 1991.

[7] G. N. Fowler, E. M. Friedlander, R. M. Weiner, and G. Wilk, "Possible manifestation of quark-gluon plasma in multiplicity distributions from high-energy reactions," Physical Review Letters, vol. 57, no. 17, pp. 2119-2122, 1986.

[8] E. M. Friedlander and R. M. Weiner, "Evidence from very large transverse momenta of a change with temperature of velocity of sound in hadronic matter," Physical Review Letters, vol. 43, pp. $15-18,1979$.

[9] M. G. Albrow, S. Almehed, P. S. L. Booth et al., "Studies of proton-proton collisions at the CERN ISR with an identified charged hadron of high transverse momentum at $90^{\circ}$ : (II) On the distribution of charged particles in the central region," Nuclear Physics B, vol. 145, no. 2-3, pp. 305-348, 1978.

[10] A. Bialas, R. A. Janik, and R. Peschanski, "Unified description of Bjorken and Landau 1+1 hydrodynamics," Physical Review C nuclear physics, vol. 76, no. 5, Article ID 054901, 2007.

[11] C. Y. Wong, "Landau hydrodynamics reexamined," Physical Review C: Nuclear Physics, vol. 78, no. 5, Article ID 054902, 2008.

[12] G. Beuf, R. Peschanski, and E. N. Saridakis, "Entropy flow of a perfect fluid in $(1+1)$ hydrodynamics," Physical Review C: Nuclear Physics, vol. 78, no. 6, Article ID 064909, 2008.

[13] Z. J. Jiang, Y. Huang, and J. Wang, "A combined model for pseudorapidity distributions in $\mathrm{p}$-p collisions at center-of-mass energies from 23.6 to $7000 \mathrm{GeV}$," Chinese Physics C: Nuclear Physics, vol. 40, no. 7, Article ID 074104, 2016.

[14] Z. W. Wang, Z. J. Jiang, and Y. S. Zhang, "The investigations of pseudorapidity distributions of final state multiplicities in $\mathrm{Au}+\mathrm{Au}$ collisions at high energies," University of Shanghai for Science and Technology, vol. 31, p. 322, 2009.

[15] N. Suzuki, "One-dimensional hydrodynamical model including phase transition," Physical Review C, vol. 81, no. 4, Article ID 044911, 2010.

[16] E. K. G. Sarkisyan and A. S. Sakharov, "Relating multihadron production in hadronic and nuclear collisions," The European Physical Journal C, vol. 70, no. 3, pp. 533-541, 2010.

[17] A. Bialas and R. Peschanski, "Asymmetric (1+1)-dimensional hydrodynamics in high-energy collisions," Physical Review C: Nuclear Physics, vol. 83, no. 5, Article ID 054905, 2011.

[18] Z.-J. Jiang, J.-Q. Hui, and H.-P. Deng, "Unified hydrodynamics and pseudorapidity distributions of charged particles produced in heavy ion collisions at low energies at RHIC," Chinese Physics Letters, vol. 34, no. 5, Article ID 052501, 2017.
[19] Z. J. Jiang, Q. G. Li, and H. L. Zhang, "Revised Landau hydrodynamic model and the pseudorapidity distributions of charged particles produced in nucleus-nucleus collisions at maximum energy at the BNL Relativistic Heavy Ion Collider," Physical Review C: Nuclear Physics, vol. 87, no. 4, Article ID 044902, 2013.

[20] C. Gale, S. Jeon, and B. Schenke, "Hydrodynamic modeling of heavy-ion collisions," International Journal of Modern Physics A, vol. 28, no. 11, Article ID 1340011, 2013.

[21] U. Heinz and R. Snellings, "Collective flow and viscosity in relativistic heavy-ion collisions," Annual Review of Nuclear and Particle Science, vol. 63, no. 1, pp. 123-151, 2013.

[22] A. N. Mishra, R. Sahoo, E. K. G. Sarkisyan, and A. S. Sakharov, "Effective-energy budget in multiparticle production in nuclear collisions," The European Physical Journal C, vol. 74, Article ID 3147, 2014.

[23] Z. J. Jiang, Y. Zhang, H. L. Zhang, and H. P. Deng, "A description of the pseudorapidity distributions in heavy ion collisions at RHIC and LHC energies," Nuclear Physics A, vol. 941, pp. 188200, 2015.

[24] H. Niemi, K. J. Eskola, and R. Paatelainen, "Event-by-event fluctuations in a perturbative QCD + saturation + hydrodynamics model: Determining QCD matter shear viscosity in ultrarelativistic heavy-ion collisions," Physical Review C, vol. 93, Article ID 024907, 2016.

[25] J. Noronha-Hostler, M. Luzum, and J.-Y. Ollitrault, "Hydrodynamic predictions for $5.02 \mathrm{TeV} \mathrm{Pb}-\mathrm{Pb}$ collisions," Physical Review C Nuclear Physics, vol. 93, no. 3, Article ID 034912, 2016.

[26] J. S. Moreland and R. A. Soltz, "Hydrodynamic simulations of relativistic heavy-ion collisions with different lattice quantum chromodynamics calculations of the equation of state," Physical Review C, vol. 93, Article ID 044913, 2016.

[27] E. K. G. Sarkisyan, A. N. Mishra, R. Sahoo, and A. S. Sakharov, "Multihadron production dynamics exploring the energy balance in hadronic and nuclear collisions," Physical Review D: Particles, Fields, Gravitation and Cosmology, vol. 93, Article ID 054046, 2016.

[28] E. K. G. Sarkisyan, A. N. Mishra, R. Sahoo, and A. S. Sakharov, "Centrality dependence of midrapidity density from $\mathrm{GeV}$ to $\mathrm{TeV}$ heavy-ion collisions in the effective-energy universality picture of hadroproduction," Physical Review D, vol. 94, Article ID 011501, 2016.

[29] K. Jiang, Y. Zhu, W. Liu et al., "Onset of radial flow in $p+p$ collisions," Physical Review C: Nuclear Physics, vol. 91, no. 2, Article ID 024910, 2015.

[30] W. Alberico, A. Lavagno, and P. Quarati, "Non-extensive statistics, fluctuations and correlations in high-energy nuclear collisions," The European Physical Journal C, vol. 12, no. 3, pp. 499-506, 2000.

[31] M. Biyajima, T. Mizoguchi, N. Nakajima, N. Suzuki, and G. Wilk, "Modified Hagedorn formula including temperature fluctuation: Estimation of temperatures at RHIC experiments," The European Physical Journal C, vol. 48, no. 2, pp. 597-603, 2006.

[32] C. Tsallis, "Possible generalization of Boltzmann-Gibbs statistics," Journal of Statistical Physics, vol. 52, no. 1-2, pp. 479-487, 1988.

[33] D. F. Torres, H. Vucetich, and A. Plastino, "Early universe test of nonextensive statistics," Physical Review Letters, vol. 79, no. 9, pp. 1588-1590, 1997. 
[34] D. B. Ion and M. L. Ion, "Entropic lower bound for the quantum scattering of spinless particles," Physical Review Letters, vol. 81, no. 26, pp. 5714-5717, 1998.

[35] A. K. Rajagopal, R. S. Mendes, and E. K. Lenzi, "Quantum statistical mechanics for nonextensive systems: Prediction for possible experimental tests," Physical Review Letters, vol. 80, no. 18, pp. 3907-3910, 1998.

[36] G. Kaniadakis, A. Lavagno, M. Lissia, and P. Quarati, "Anomalous diffusion modifies solar neutrino fluxes," Physica A: Statistical Mechanics and its Applications, vol. 261, no. 3-4, pp. 359-373, 1998.

[37] A. R. Plastino and A. Plastino, "Information theory, approximate time dependent solutions of Boltzmann's equation and Tsallis' entropy," Physics Letters A, vol. 193, no. 3, pp. 251-258, 1994.

[38] U. Tirnakli, F. Bykkilic, and D. Demirhan, "Some bounds upon the nonextensivity parameter using the approximate generalized distribution functions," Physics Letters A, vol. 245, no. 1-2, pp. 62-66, 1998.

[39] S. Grigoryan, "Using the Tsallis distribution for hadron spectra in $p p$ collisions: pions and quarkonia at $\sqrt{s}=5-13000 \mathrm{Gev}$," Physical Review D: Particles, Fields, Gravitation and Cosmology, vol. 95, Article ID 056021, 2017.

[40] D. Thakur, S. Tripathy, P. Garg, R. Sahoo, and J. Cleymans, "Indication of a differential freeze-out in proton-proton and heavy-ion collisions at RHIC and LHC energies," Advances in High Energy Physics, vol. 2016, Article ID 4149352, 13 pages, 2016.

[41] J.-Q. Hui, Z.-J. Jiang, and D.-F. Xu, "A description of the transverse momentum distributions of charged particles produced in heavy ion collisions at RHIC and LHC energies," Advances in High Energy Physics, vol. 2018, Article ID 7682325, 9 pages, 2018.

[42] J. Cleymans and D. Worku, "The Tsallis distribution in proton-proton collisions at $\sqrt{s}=0.9 \mathrm{TeV}$ at the LHC," Journal of Physics G: Nuclear and Particle Physics, vol. 39, no. 2, Article ID 025006, 2012.

[43] F. Cooper and G. Frye, "Landau's hydrodynamic model of particle production and electron-positron annihilation into hadrons," Physical Review D, vol. 11, Article ID 192, 1975.

[44] B. I. Abelev, J. Adams, and M. M. Aggarwal, "Strange particle production in $p+p$ collisions at $\sqrt{s}=200 \mathrm{GeV}$," Physical Review C: Nuclear Physics, vol. 75, Article ID 64901, 2007.

[45] A. Adare, S. Afanasiev, and C. Aidala, "Identified charged hadron production in $p+p$ collisions at $\sqrt{s}=200$ and $62.4 \mathrm{GeV}$," Physical Review C: Nuclear Physics, vol. 83, Article ID 064903, 2011.

[46] S. Chatrchyan, V. Khachatryan, and A. M. Sirunyan, "Study of the inclusive production of charged pions, kaons, and protons in pp collisions at $\sqrt{s}=0.9,2.76$, and $7 \mathrm{TeV}$, The European Physical Journal C, vol. 72, Article ID 2164, 2012.

[47] A. Adare, S. Afanasiev, and C. Aidala, "Scaling Properties of Azimuthal Anisotropy in $\mathrm{Au}+\mathrm{Au}$ and $\mathrm{Cu}+\mathrm{Cu}$ Collisions at $\sqrt{s_{N N}}=200 \mathrm{GeV}$," Physical Review Letters, vol. 98, Article ID 162301, 2007.

[48] F.-H. Liu, T. Tian, J.-X. Sun, and B.-C. Li, "What can we learn from (Pseudo) rapidity distribution in high energy collisions?" Advances in High Energy Physics, vol. 2014, Article ID 863863, 10 pages, 2014.

[49] D. Teaney, J. Lauret, and E. V. Shuryak, "Flow at the SPS and RHIC as a quark-gluon plasma signature," Physical Review Letters, vol. 86, no. 21, pp. 4783-4786, 2001.
[50] M. Strickland, "Thermal $\Upsilon(1 s)$ and $\chi_{b 1}$ suppression at $\sqrt{ }\left(s_{N N}\right)=$ 2.76 TeV Pb-Pb Collisions at the LHC," Physical Review Letters, vol. 107, Article ID 132301, 2011.

[51] B. I. Abelev, M. M. Aggarwal, and Z. Ahammed, "Systematic measurements of identified particle spectra in $p p, d+A u$, and $\mathrm{Au}+\mathrm{Au}$ collisions at the STAR detector," Physical Review C: Nuclear Physics, vol. 79, no. 3, Article ID 034909, p. 58, 2009.

[52] H. L. Lao, F. H. Liu, and R. A. Lacey, "Extracting kinetic freezeout temperature and radial flow velocity from an improved Tsallis distribution," The European Physical Journal A, vol. 53, p. 44, 2017. 

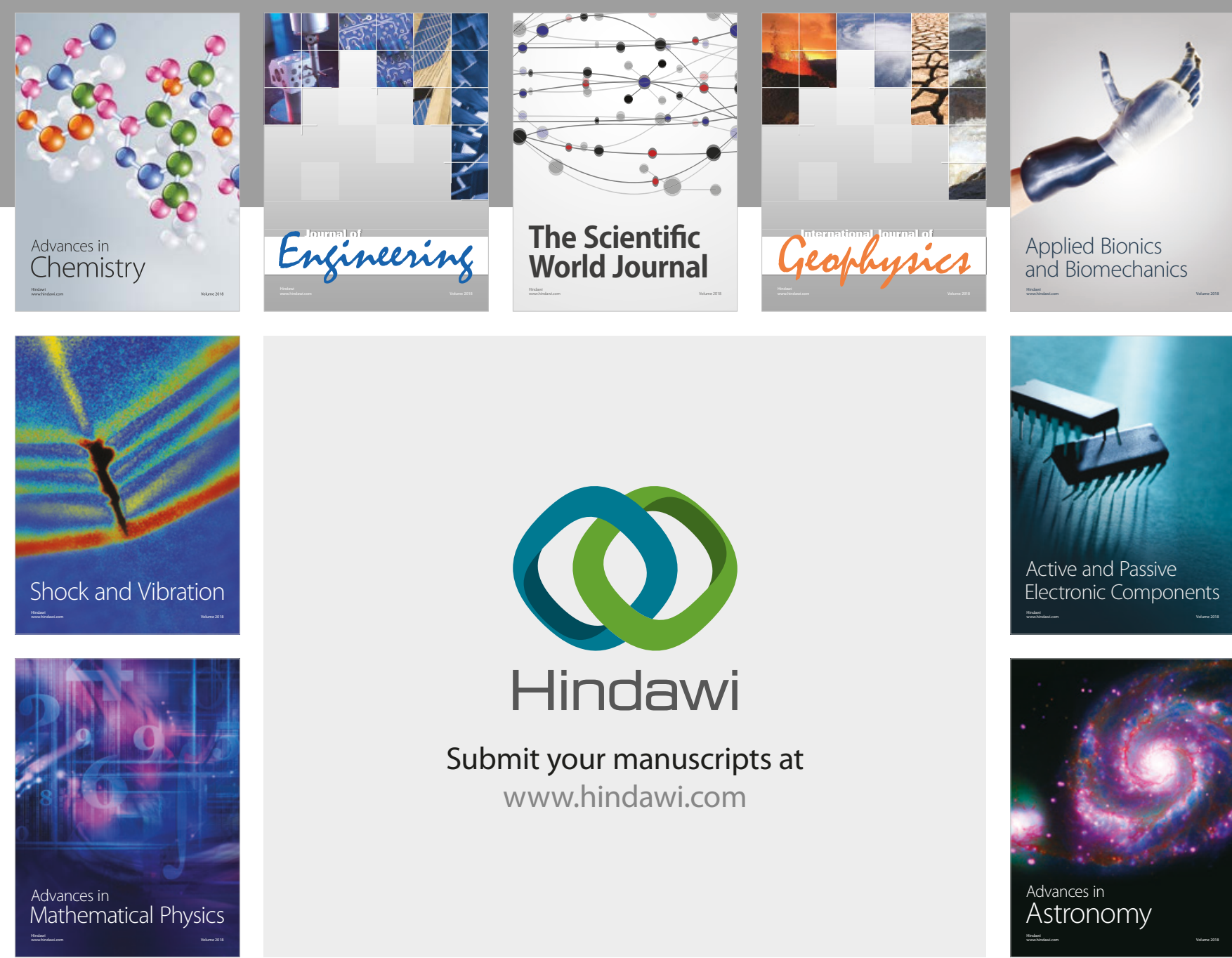

Submit your manuscripts at

www.hindawi.com

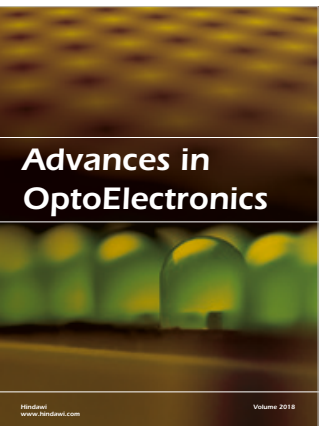

\section{Rotcting Machinery}
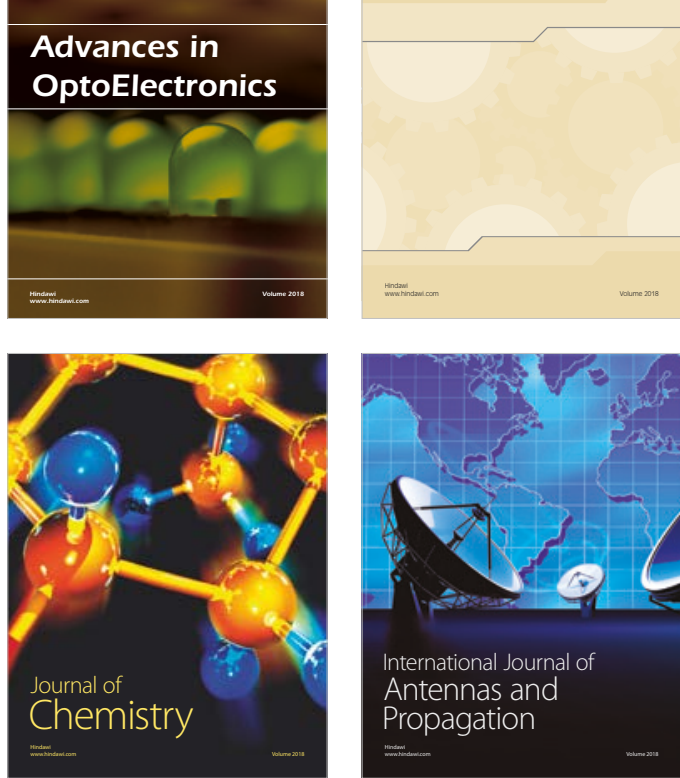

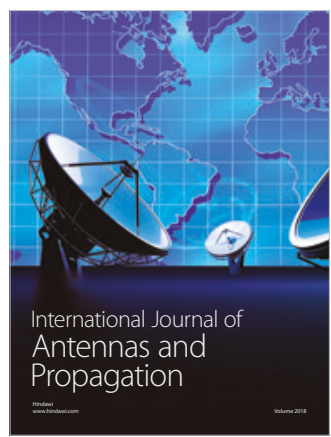

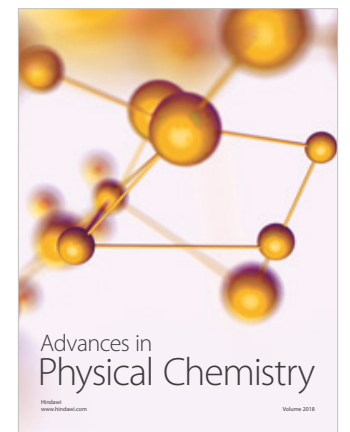

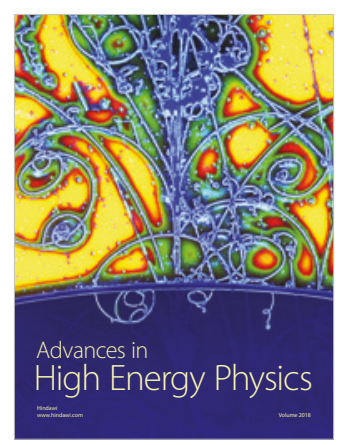

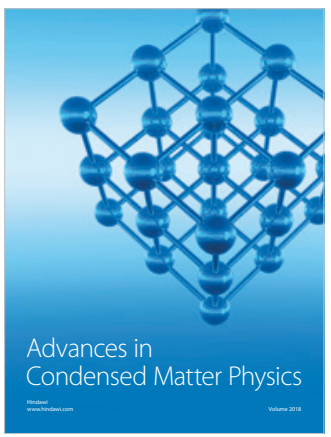

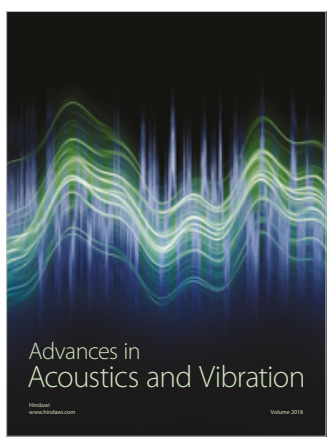

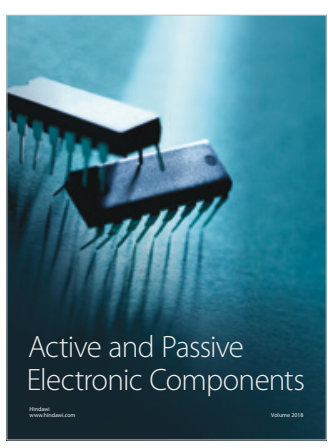
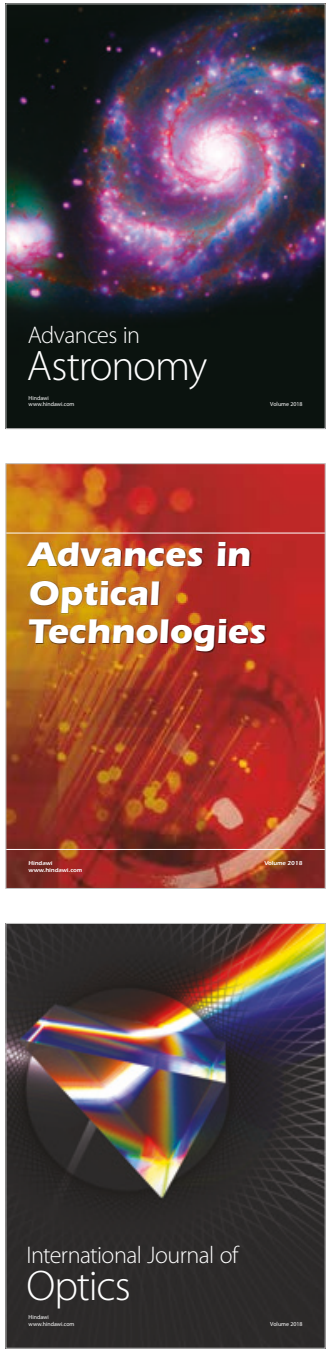